\title{
Treatment of tardive dyskinesia: A systematic review (1997-2011)
}

\section{Alimi ${ }^{\star, \star *}$ \\ P. Gaillard ${ }^{*, * * *}$ \\ V. Camus ${ }^{\star, \star * \star}$ \\ W. El-Hage $\mathrm{e}^{\star, \star \star \star}$}

* Clinique Psychiatrique Universitaire, CHRU de Tours

** Centre Hospitalier Esquirol, CHU de Caen

*** Inserm U930 ERL, Université François Rabelais de Tours

FRANCE

\begin{abstract}
Background and Objectives: Tardive dyskinesia (TD) is a frequent and incapacitating side effect of first-generation antipsychotics. Although second-generation antipsychotics (SGAs) seem to be associated with a decreased risk of TD, it remains a severe, unresolved iatrogenic condition. Moreover, there is no commonly accepted effective treatment for TD.

We conducted a systematic review of the literature to assess evidence regarding the effectiveness of different therapeutic interventions for TD.

Methods: We performed a systematic review focussing exclusively on randomised controlled trials (RCTs). We searched the MEDLINE database (1997 to 2011) using the keyword "tardive dyskinesia" within the "title" search field. Twenty-six RCTs were included. Based on the evidence from RCTs, we built a decision tree that healthcare professionals can use to choose an effective therapeutic intervention for TD.

Results: Four therapeutic interventions were found to be effective in TD (vitamin B6, ginkgo biloba, branched-chain amino acids, and piracetam).

Conclusions: Patients with TD could benefit from the therapeutic interventions supported by the data accumulated from RCTs.
\end{abstract}

Received: 27 February 2012

Revised: 4 January 2013

Accepted: 15 April 2013 


\section{Background}

Tardive dyskinesia (TD) causes severe social and physical disability. TD is characterised by abnormal involuntary movements of the tongue, jaw, trunk, or extremities that persist for at least 4 weeks. The symptoms appear during neuroleptic treatment or within 4 weeks after discontinuing neuroleptic treatment that has been administered for at least 3 months. The annual incidence of TD varies between 4 and $5 \%$ in patients using first-generation antipsychotics (FGAs) ${ }^{1}$. The use of second-generation antipsychotics (SGAs) is associated with a reduced rate of $\mathrm{TD}^{2}$, with an estimated incidence ranging from 2.1 to $4.9 \%{ }^{3,4}$.

Primary prevention that prevents the disease from occurring remains the best strategy. In the case of $\mathrm{TD}$, the lowest effective dose should be used ${ }^{5,6}$ for the shortest amount of time possible ${ }^{7}$. There is also a class effect: SGAs cause fewer cases of dyskinesia ${ }^{4}$, and clozapine has not shown any correlation to $\mathrm{TD}^{8}$. Moreover, prescribing anticholinergic therapy in patients with acute extrapyramidal symptoms is a risk factor for $\mathrm{TD}^{7}$.

Currently, there is no evidence supporting the effectiveness of any therapeutic intervention for this illness ${ }^{9}$. Nevertheless, several publications have reported an alleviating effect of some treatments for TD symptoms. This review aims to determine the effectiveness and safety of using these proposed therapeutic interventions for TD.

\section{Objectives}

This review aims to determine the effects of any intervention for neuroleptic-induced TD in people with schizophrenia or other chronic mental illness.

\section{Methods}

\section{Criteria for considering studies for this review}

Types of studies. We included only randomised controlled trials (RCTs) described as single- or double-blind.

Types of participants. We included people suffering from schizophrenia or chronic mental illness, irrespective of diagnosis criteria used, who required neuroleptic medications and developed neuroleptic-induced TD.

Types of interventions. We included trials whose aim was to assess any type of intervention in neuroleptic induced TD.

Types of outcome measures. This paper reviewed four health outcome measures that were commonly used in the selected RCTs to assess changes in patients. To determine clinical relevance, we defined significant change using the original cut-off point chosen by the authors.

- Tardive dyskinesia: No significant change in TD score; average endpoint TD score; average change in TD score.

- Mental state: No significant change in mental state; average endpoint mental state score; average change in mental state score.

- Adverse effects: Clinically important adverse effects; any adverse effect.

- Leaving the study early for general reasons.

\section{Search method for identification of studies}

A comprehensive systematic literature review was undertaken using electronic database search engines. We searched the MED- 
LINE database for articles published between 1997 and 2011 using the keyword "tardive dyskinesia" in the "title" search field. We considered all of the clinical trials returned by this search.

\section{Data collection and analysis}

Selection of studies. Two of the authors (MA, WEH) independently and manually inspected the search results to retrieve those studies that were likely relevant. Where disagreement existed, we tried to resolve it by discussion. If a disagreement could not be resolved, we included the trial with a description of the ambiguity in the results section.

Data extraction and management. Two authors (MA, WEH) extracted data independently. In cases of disagreement, we tried to achieve resolution via discussion. If doubt persisted, we did not use the data and mentioned the lack of information. We assessed outcomes using quantitative (i.e., TD score change) or qualitative data (no significant change or significant change in TD score). When it was possible, we used binary data. Participants were divided into "clinically improved" or "not clinically improved" according to the primary cut-off point presented by the authors.

\section{Assessment of risk of bias in included studies}

The various risks of bias were evaluated as low, high, or uncertain, using the tool published in the Cochrane Collaboration Handbook ${ }^{10}$. For assessing the risk of bias, this tool recommends that evaluators pay attention to the sequence generation, the allocation concealment, the blinding of participants, the completeness of information, and the risk of selective reporting.

\section{Measures of treatment effect}

For continuous data, we calculated the effect size and the $95 \%$ confidence interval according to Hedges and Olkin's formula ${ }^{11}$. When binary data were presented, we calculated a relative risk and its $95 \%$ confidence interval $^{12}$. When results were significant, we used the tool provided in Grade Profiler 3.6 software (ims.cochrane.org) to determine the absolute risk reduction (ARR) and its 95\% confidence interval. The number needed to treat (NNT) and its 95\% confidence interval were calculated as the inverse of the ARR.

\section{Unit of analysis issues}

Some studies used randomisation by clusters, leading to a significant risk of type I error, reducing the level of evidence provided by the test. In crossover trials, there is a risk of a persistent effect between the two phases despite the wash-out period. This risk is reasonable in the case of chronic patients in stable condition, in an attempt to reduce variance and to increase effective sample size.

\section{Dealing with missing data}

The rate of loss affects the credibility of published data. We excluded data from studies where more than $50 \%$ of the participants in any group were lost to follow-up.

\section{Results}

\section{Selected references}

Our search of the MEDLINE database returned 81 references; among these, only 41 articles described therapeutic interventions for symptoms of TD. After controlling for the inclusion criteria, this review included a to- 
Table 1

Description of the 26 included and 15 excluded studies

\begin{tabular}{|c|c|c|c|c|c|}
\hline Studies & Year & $\mathrm{N}$ & Age (years) & Length & Intervention evaluated \\
\hline \multicolumn{6}{|l|}{ Included studies } \\
\hline Dorevitch et al. (25) & 1997 & 40 & 64,4 & 8 weeks & Vitamin E \\
\hline Sajjad (26) & 1998 & 20 & $67,8^{1}$ & 7 months & Vitamin E \\
\hline Adler et al. (27) & 1998 & 40 & $61,1^{1}$ & 8 weeks & Vitamin E \\
\hline Adler et al. (28) & 1999 & 158 & $50,7^{1}$ & 12 months & Vitamin E \\
\hline Zhang et al. (29) & 2004 & 41 & $54,5^{1}$ & 12 weeks & Vitamin E \\
\hline Hayashi et al. (30) & 1997 & 38 & $64,6^{2} / 63,7^{2}$ & 5 weeks & Mianserine / trazodone \\
\hline Angus et al. (31) & 1997 & 16 & 65 & 3 weeks & Amantadine \\
\hline Pappa et al. (32) & 2010 & 22 & 52 & 2 weeks & Amantadine \\
\hline Cowen et al. (33) & 1997 & 33 & $38^{3} / 77,3^{3}$ & 3 weeks & Acetazolamide + thiamine \\
\hline Mosnik et al. (34) & 1997 & 18 & 44,1 & 1 day & Phenylalanine \\
\hline Shamir et al. (35) & 2000 & 19 & 74 & 4 weeks & Melatonin \\
\hline Shamir et al. (36) & 2001 & 22 & 64,2 & 6 weeks & Melatonin \\
\hline Lerner et al. (37) & 2001 & 15 & $28-71^{4}$ & 4 weeks & Vitamin B6 \\
\hline Lerner et al. (38) & 2007 & 50 & 47 & 4 weeks & Vitamin B6 \\
\hline Wonodi et al. (39) & 2004 & 14 & 47 & 4 weeks & Naltrexone \\
\hline Bai et al. (40) & 2003 & 49 & 50,2 & 12 weeks & Risperidone \\
\hline Emsley et al. (41) & 2004 & 45 & 49,7 & 50 weeks & Quetiapine/Haloperidol \\
\hline Chan et al. (42) & 2010 & 60 & $42,7^{5} / 48^{5}$ & 24 weeks & Risperidone/Olanzapine \\
\hline Richardson et al. (43) & 2003 & 52 & 48,0 & 3 weeks & Branched-chain amino acids \\
\hline Emsley et al. (44) & 2006 & 84 & 42,9 & 12 weeks & Eicosapentanoic acid \\
\hline Caroff et al. (45) & 2007 & 35 & 56,4 & 12 weeks & Galantamine \\
\hline Libov et al. (46) & 2007 & 40 & 47 & 4 weeks & Piracetam \\
\hline Woods et al. (47) & 2008 & 50 & $45,1^{1}$ & 12 weeks & Levetiracetam \\
\hline Ogunmefun et al. (48) & 2009 & 7 & 62,2 & 4 weeks & Donepezil \\
\hline Zhang et al. (49) & 2011 & 157 & 45,3 & 12 weeks & Ginkgo biloba \\
\hline Damier et al. (50) & 2007 & 10 & 45,1 & & Deep brain stimulation \\
\hline \multicolumn{6}{|l|}{ Excluded studies } \\
\hline Spivak et al. (51) & 1997 & 20 & 43,1 & 18 weeks & Clozapine \\
\hline Bassitt\&Louza Neto (52) & 1998 & 7 & 28,5 & 24 weeks & Clozapine \\
\hline Kinon et al. (53) & 2004 & 95 & 48,5 & 8 months & Olanzapine \\
\hline Bai et al. (54) & 2005 & 40 & 49,6 & 48 weeks & Risperidone \\
\hline Lerner et al. (55) & 1999 & 5 & NS & 4 weeks & Vitamin B6 \\
\hline Ondo et al. (56) & 1999 & 20 & 65,2 & 3 months & Tetrabenazine \\
\hline Sirota et al. (57) & 2000 & 20 & 69,8 & 12 weeks & Ondansetron \\
\hline Michael et al. (58) & 2002 & 6 & 57,8 & 1 month & Vitamins E \& C \\
\hline Hardoy et al. (59) & 2003 & 30 & 49,7 & 1 year & Gabapentin \\
\hline Richardson et al. (60) & 2004 & 6 & 13 & 2 weeks & Branched-chain amino acids \\
\hline Bona (61) & 2006 & 17 & 50 & 6 months & Levetiracetam \\
\hline Meco et al. (62) & 2006 & 16 & 69 & 3 months & Levetiracetam \\
\hline Konitsiotis et al. (63) & 2006 & 8 & 55,1 & 1 month & Levetiracetam \\
\hline Lee et al. (64) & 2007 & 69 & 49 & 16 weeks & Kamishoyosan \\
\hline Miyaoka et al. (65) & 2008 & 22 & 57,1 & 12 weeks & Yi-gan-san \\
\hline
\end{tabular}

\footnotetext{
${ }^{1}$ Treatment group $;{ }^{2}$ Mianserin vs. trazodone groups; ${ }^{3}$ Younger vs. older patient groups; ${ }^{4}$ Range;
}

${ }^{5}$ Risperidone/olanzapine groups. 
tal of 26 references. We excluded 15 studies due to a lack of randomisation protocol.

Design. Fourteen trials used a crossover design; 12 were conducted in parallel groups.

Participants. Participants suffered from psychiatric disorders, most often schizophrenia or schizoaffective disorder (18 trials).

Study location. Most of the studies were carried out exclusively among inpatients (17 trials).

Outcomes. The studies often reported outcomes in the form of scale-derived scores. TD was assessed using three different scales: the Abnormal Involuntary Movement Scale $(\text { AIMS })^{13}$, the dyskinesia subscale of the Extrapyramidal Symptom Rating Scale (ESRS) ${ }^{14}$, and the Maryland Psychiatric Research Center Involuntary Movement Scale (MPRC) ${ }^{15}$. Other extrapyramidal symptoms were assessed using the ESRS, the Simpson-Angus Scale (SAS) ${ }^{16}$, and the Barnes Akathisia Rating Scale $(\mathrm{BARS})^{17}$. Mental state was assessed using the Brief Psychiatric Rating Scale (BPRS) ${ }^{18}$ and the Positive and Negative Syndrome Scale (PANSS) ${ }^{19}$. Other tests used included the Global Assessment of Functioning scale $(\mathrm{GAF})^{20}$, the Mini Mental State Examination (MMSE) ${ }^{21}$, the Auditory Verbal Learning Test ${ }^{22}$, the Continuous Performance Test $^{23}$, and the Stroop test ${ }^{24}$.

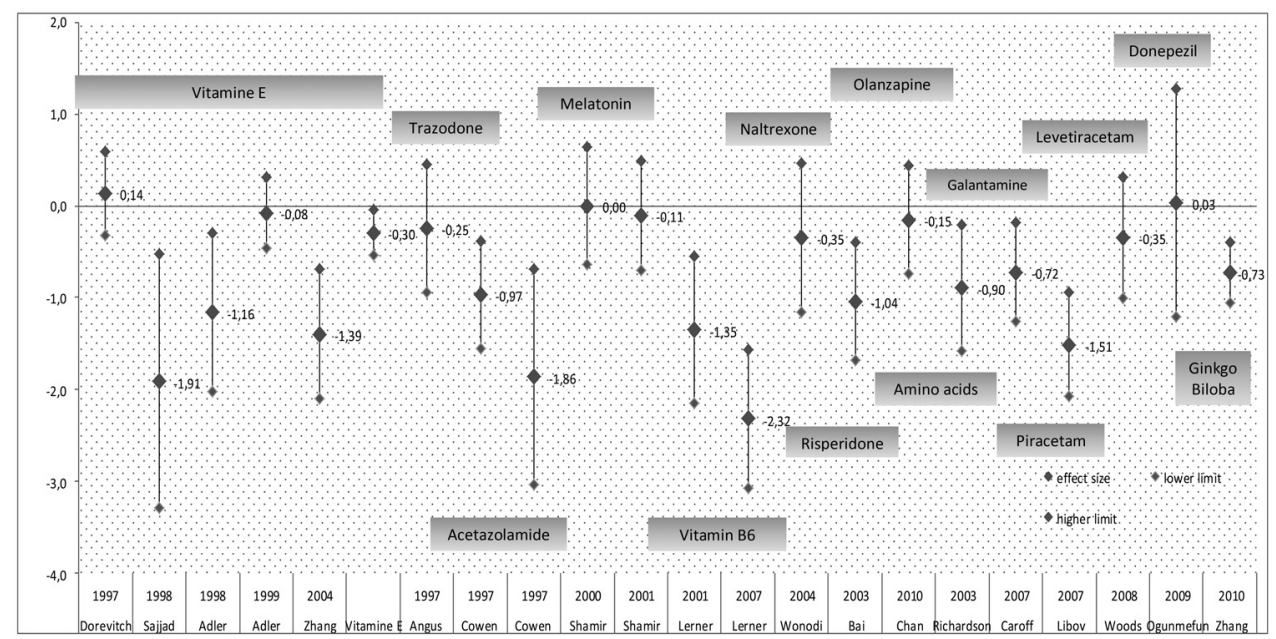

Figure 1. Graphical representation of effect sizes (Hegde's g).

\section{Risk of bias in included studies}

Among the selected RCTs, only three studies ${ }^{29,46,55}$ concealed the patient allocation. Two active trials had a single-blind design ${ }^{41,42}$; the remaining studies used a double-blind de- sign, although their methods were not always described. The data from the studies often allowed us to calculate the relative risk of leaving the study early. We were not able to use data from two of the studies due to poor presentation $^{30,32}$. 


\section{Effects of interventions}

The efficacy of the different interventions in treating the symptoms of TD is summarised in Tables 2 and 3. Three studies supported the efficacy of vitamin $E^{26,27,29}$. We found evidence for a modest efficacy of vitamin $\mathrm{E}$ in the TD scores (five trials, $\mathrm{n}=249$, Hedge's g effect size $(\mathrm{ES})=-0.30,95 \% \mathrm{CI}=$ -0.54 to -0.05$)$. We did not find any evidence supporting the efficacy of amantadine, phenylalanine, melatonin, naltrexone, eicosapentanoic acid, donepezil, or levetiracetam in the TD scores. The published data concerning mianserin and trazodone were poorly presented and unusable. We found a significant difference in the average endpoint TD scores for acetazolamide, thiamine, vitamin B6, branched-chain amino acids, galantamine, piracetam, and ginkgo biloba.

Three trials assessed the action of SGAs in TD. Risperidone was compared to a placebo ${ }^{40}$ and olanzapine ${ }^{42}$, and quetiapine was compared to haloperidol ${ }^{41}$. We found a significant difference between the average endpoint AIMS scores of risperidone and placebo (ES $=-1.09,95 \% \mathrm{CI}=-0.39$ to -0.96$)$. Risperidone and olanzapine had significantly different effects $(p=0.55)$, but they did not produce different clinical improvements in $\mathrm{TD}(\mathrm{RR}=0.84,95 \% \mathrm{CI}=0.55$ to 1.30$)$. Quetiapine did not show a clinical improvement compared with haloperidol $(\mathrm{RR}=0.64$, $95 \% \mathrm{CI}=0.31$ to 1.32 ).

One study evaluated the efficacy of bilateral pallidal stimulation in resistant $\mathrm{TD}^{50}$. The two conditions of stimulation (on-off) were applied to patients over 2 days under doubleblind conditions. The authors did not publish the results obtained for blind conditions.

Mental state. Mental state was evaluated by the BPRS in five studies $28,30,40,42,45$, the PANSS in three studies ${ }^{41,44,49}$, and the MMSE in one study ${ }^{45}$. This review did not find any significant results from additional treatments on psychiatric symptomatology.

Adverse effects. Extrapyramidal symptoms were evaluated by the BARS in two studies $^{28,45}$, the SAS in three studies ${ }^{28,33,45}$, and the ESRS in four studies ${ }^{37,38,40,46}$. The improvement in parkinsonism was significant for vitamin B6 at doses of $400 \mathrm{mg}(\mathrm{ES}=-1.58$, $95 \% \mathrm{CI}=-2.36$ to -0.73$)^{37}$ and $1200 \mathrm{mg}(\mathrm{ES}$ $=-7.61,95 \% \mathrm{CI}=-9.13$ to 5.83$)^{38}$. There was a significant worsening of extrapyramidal symptoms with galantamine $(\mathrm{ES}=3.33,95 \%$ $\mathrm{CI}=2.50$ to 4.08$)$. There was no difference in akathisia scores $(\mathrm{ES}=0,95 \% \mathrm{CI}=-0.52$ to 0.52$)$. In all other cases, the extrapyramidal symptomatology remained unchanged. No other severe adverse effects were found in this review.

Leaving the study early. The risk of leaving the study early was noted in most articles included in this review. This risk was not significantly different among the groups included in each trial.

\section{Discussion}

\section{Summary of main results}

This review suggests that there are scant evidence-based data supporting the efficacy of pharmacological or non-pharmacological interventions for treating TD.

By modifying the balance of dopamine and serotonin in the basal ganglia, 5HT2A antagonism is expected to reduce the symptoms of dyskinesia ${ }^{66,67}$. SGAs showed superior efficacy to placebos ${ }^{40}$, but not to FGAs, for this indication $^{41}$. Moreover, an improvement with risperidone was maintained over a period of 48 weeks under open-label conditions ${ }^{54}$. 


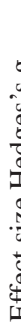

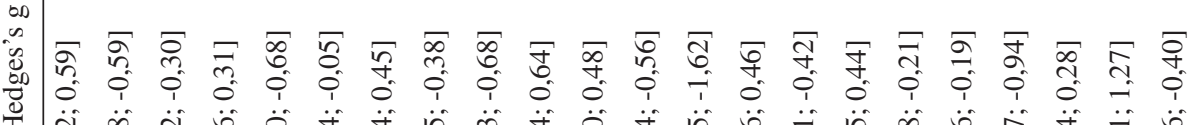

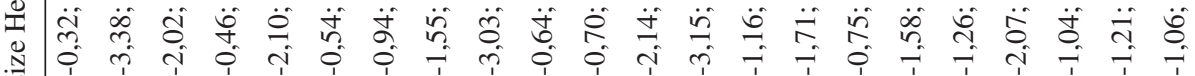

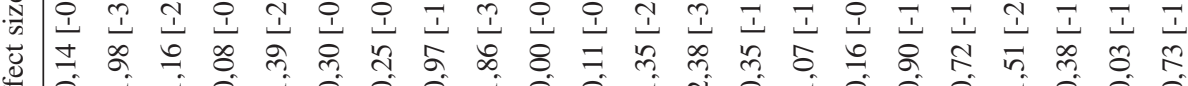

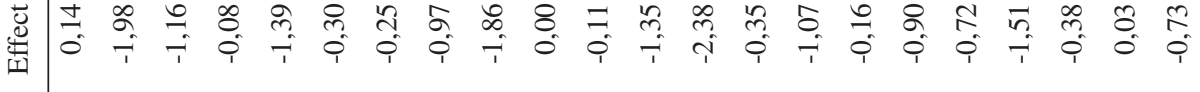

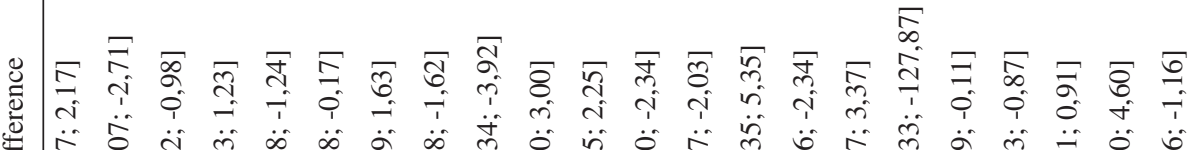

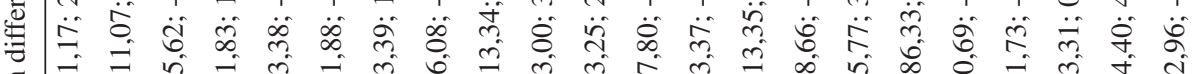

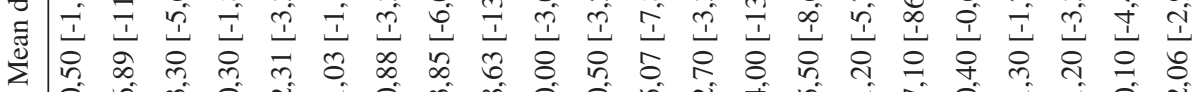

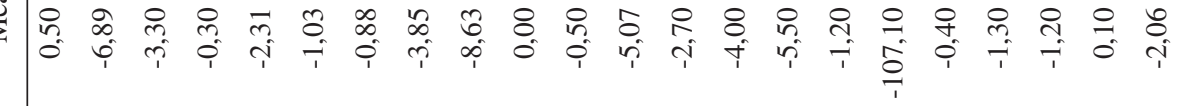

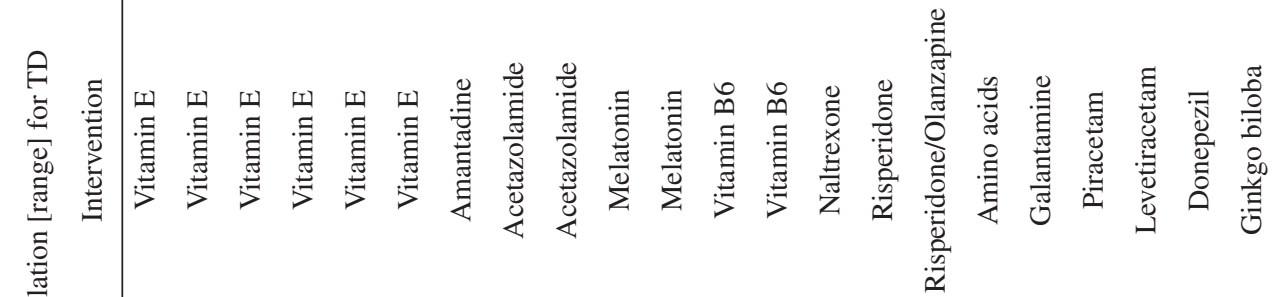

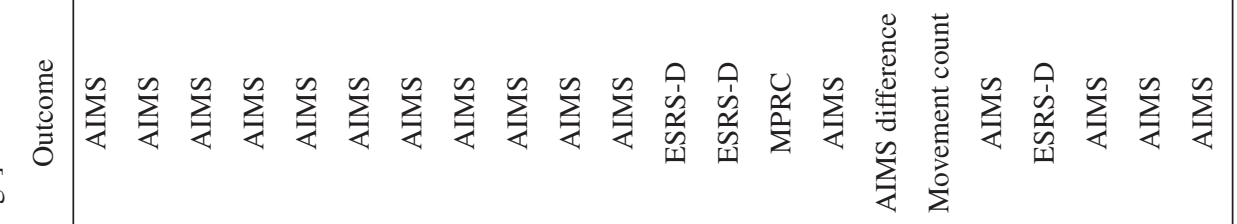

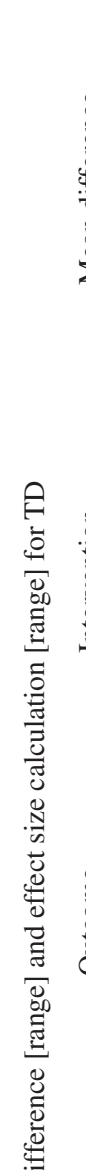

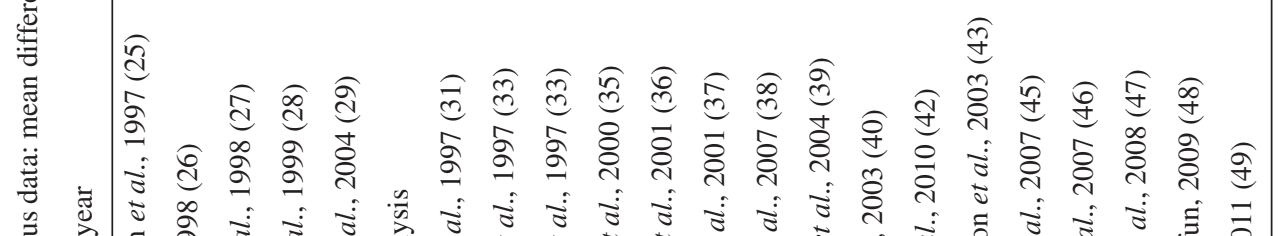

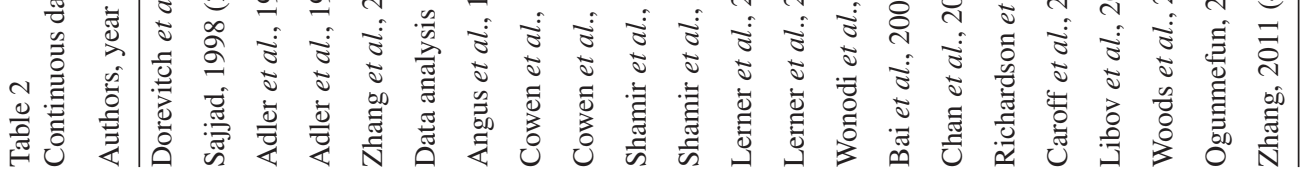




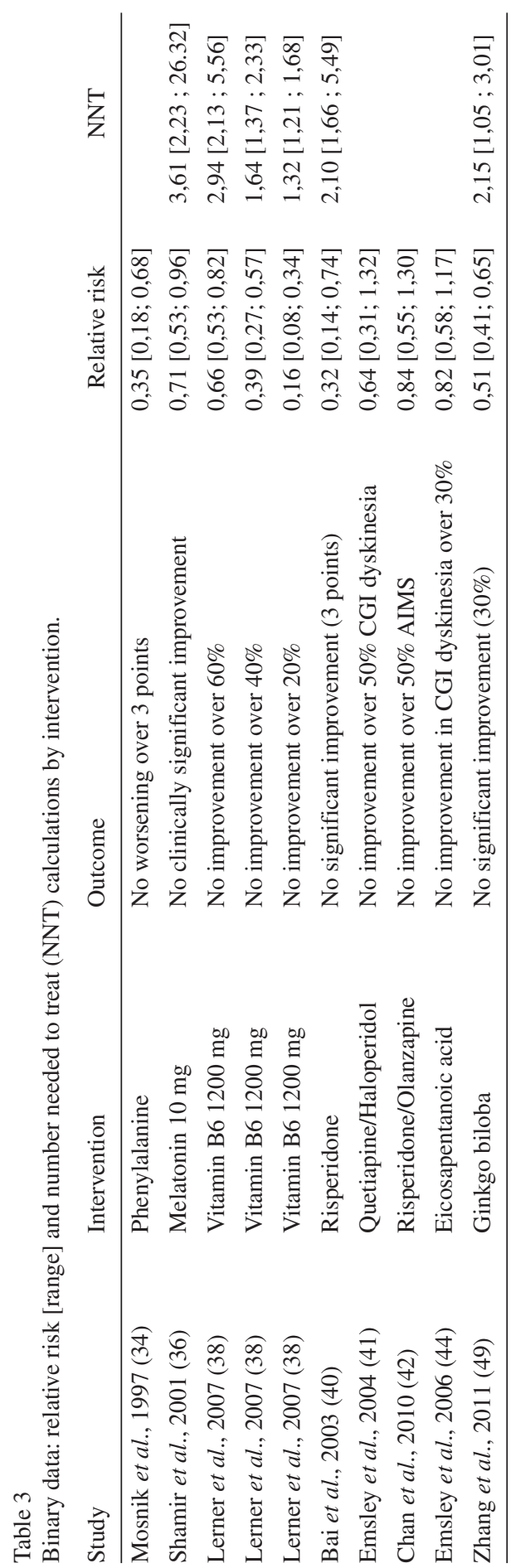

We found a positive effect of branchedchain amino acids in one study ${ }^{43}$. This intervention appears safe but is supported by lowquality evidence.

We found evidence for the efficacy of piracetam for TD and parkinsonian symptoms in one study ${ }^{46}$. We assessed the evidence provided by this study as low quality. Thus, piracetam, whose safety is well established, might be a reasonable treatment for TD.

Two trials reported the efficacy of vitamin B6 in reducing the symptoms of $\mathrm{TD}^{37,38}$. At a dose of $1200 \mathrm{mg} /$ day, the results were significant, regardless of the threshold used (AIMS score improvement of $60 \%, 40 \%$, or $20 \%)$. The NNT to obtain an improvement of $60 \%$ was estimated at 2.88 . Therefore, vitamin B6 appears to be a treatment supported by low-quality evidence.

Ginkgo biloba was found to be effective in treating the symptoms of TD in one study ${ }^{49}$ with medium-quality evidence. The NNT to obtain greater than $30 \%$ symptomatic improvement was calculated at 2.16 subjects. Thus, ginkgo biloba offers a positive benefitto-risk ratio in TD.

Only a modest benefit of vitamin E in TD has been shown, with contradictory results from different studies. Furthermore, a previous review did not find a clinically significant improvement in patients treated with vita$\min E^{68}$. Thus, we are not able to recommend the use of vitamin E for TD.

One trial determined acetazolamide to be effective in treating $\mathrm{TD}^{33}$. The quality of evidence was very low due to the very small sample size. Thus, the efficacy of acetazolamide is not supported by a sufficient amount of reliable data.

Melatonin was not found to be effective at a dose of $2 \mathrm{mg} /$ day and provided ambiguous results at $10 \mathrm{mg} /$ day. In our opinion, the data 
provided do not support a positive effect of melatonin on TD.

One study found a modest efficacy of galantamine for $\mathrm{TD}^{45}$, which was offset by its deleterious effect on extrapyramidal symptoms. Galantamine does not present a positive benefit-to-risk ratio in TD.
Vitamin B6, ginkgo biloba, piracetam, and branched-chain amino acids have shown significantly greater improvement than placebos in RCTs. The use of ginkgo biloba is supported by medium-quality evidence, while the level of evidence supporting branched-chain amino acids, piracetam, and vitamin B6 is low.

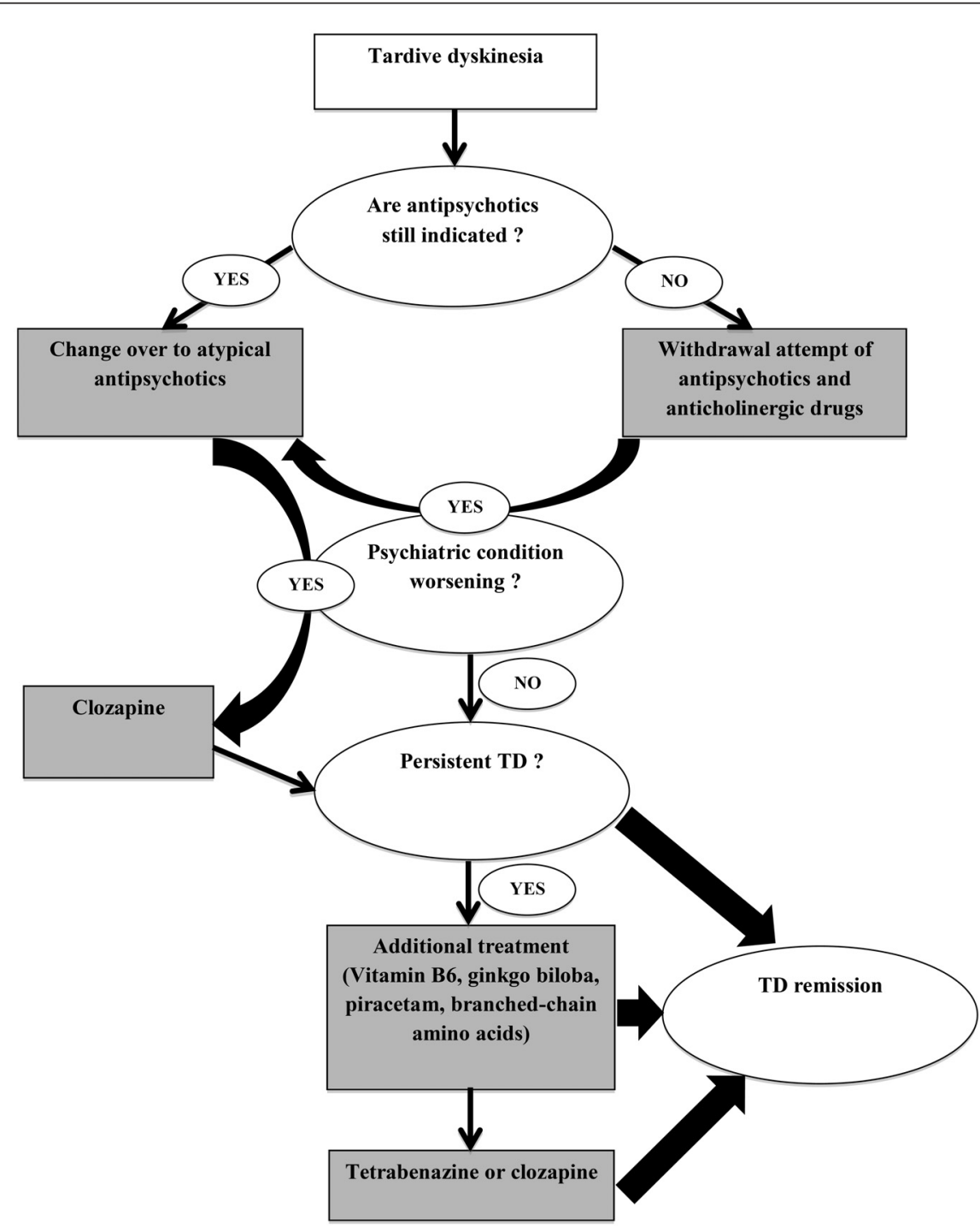

Figure 2. Decision tree for the treatment of tardive dyskinesia. 


\section{Treatment strategy for tardive dyskinesia}

In light of the data reported above, a medical decision-making pattern can be proposed. It seems necessary to reassess the indications of drug prescriptions and the risk-benefit ratios in the context of iatrogenic disease. Several guidelines recommend the reduction of antipsychotics ${ }^{1,69,70}$, though this strategy has not been proven effective in $\mathrm{TD}^{71}$. When antipsychotic drugs are definitively indicated, a treatment that is less likely to induce TD should be chosen as the firstline treatment. Risperidone and olanzapine showed comparable efficacy in this indication $^{40,42,54}$. Quetiapine did not demonstrate significantly greater efficacy than haloperi$\mathrm{dol}^{41}$. Clozapine remains a second-line treatment due to the lack of RCTs.

Four treatments evaluated in this review in addition to antipsychotic medications have shown relative efficacy for the symptoms of TD. Vitamin B6, ginkgo biloba, piracetam, and branched-chain amino acids can all be used in cases of persistent dyskinesia after a disabling relay by SGAs or neuroleptic discontinuation.

The known risk of peripheral neuropathy with high doses of pyridoxine requires longterm monitoring. Nevertheless, the authors did not describe any adverse events for a dose of $1200 \mathrm{mg}$ for 12 weeks $^{38}$. Furthermore, because the safety of the prolonged use of doses below $500 \mathrm{mg}$ has been established, vitamin B6 may be recommended at this dose in cases of persistent symptoms ${ }^{72}$. The use of ginkgo biloba, piracetam, and branched-chain amino acids does not present any particular risk.

In cases of insufficient effects of one or more of these treatments, other therapeutic options that have not been evaluated in RCTs are available. Tetrabenazine, which is already used for several movement disorders, and clozapine are commonly used by physicians in clinical practice. Some uncontrolled studies have reported the efficacy of tetrabenazine in $\mathrm{TD}^{73}$.

Considering the data presented in this review, we propose a decision tree to help clinicians choose a treatment for patients suffering from TD.

\section{Limitations}

Since the description of TD in $1957^{74}$, no effective treatment has been clearly identified. This problem remains important despite the prescription of SGAs at lower doses. The studies included in this review provide a level of evidence that is low to moderate, and they suffer from crucial limitations and imprecision. Each trial included only a small number of participants, and their results have not been reproduced.

Many treatments used experimentally in TD have not been evaluated by RCTs. The use of clozapine and tetrabenazine is based on empirical data and consistent aetiopathogenic hypotheses. These treatments, prescribed by many physicians in cases of TD, have not yet been evaluated by RCTs. Despite the fact that the neuropathology of TD remains complex and has not been fully elucidated, several theories have been proposed ${ }^{75}$ that could lead to the development of new compounds useful in the treatment of TD.

Future research on these treatments, including clozapine and tetrabenazine, could bring about significant changes in TD treatment strategies.

\section{Conclusion}

This systematic review of the literature has identified several interventions that could 
be useful in the treatment of antipsychotic-induced TD. SGAs appear to be a consistent first-line intervention for patients requiring long-term antipsychotic treatment. However, no study has demonstrated their superiority compared to FGAs in TD. Several additional treatments can benefit patients with TD (vitamin B6, piracetam, branched-chain amino acids, ginkgo biloba). In view of these results, it seems reasonable to offer symptomatic treatment to patients suffering from TD. Future research will be necessary to confirm these results and to evaluate other compounds already prescribed by physicians for TD.

\section{References}

1. American Psychiatric Association. Task Force on Tardive dyskinesia. Tardive Dyskinesia: A Task Force Report of the American Psychiatric Association. Washington, DC: American Psychiatric Association; 1992.

2. Tenback DE, van Harten PN, van Os J. Non-therapeutic risk factors for onset of tardive dyskinesia in schizophrenia: a meta-analysis. Mov Disord 2009; 24(16): 23092315 .

3. Correll CU, Leucht S, Kane JM. Lower risk for tardive dyskinesia associated with second-generation antipsychotics: a systematic review of 1-year studies. Am J Psychiatry 2004; 161(3): 414-425.

4. Correll CU, Schenk EM. Tardive dyskinesia and new antipsychotics. Curr Opin Psychiatry 2008; 21(2): 151-156.

5. Chakos MH, Alvir JM, Woerner MG, Koreen A, Geisler S, Mayerhoff D, et al. Incidence and correlates of tardive dyskinesia in first episode of schizophrenia. Arch Gen Psychiatry 1996; 53(4): 313-319.

6. Morgenstern H, Glazer WM. Identifying risk factors for tardive dyskinesia among long-term outpatients maintained with neuroleptic medications. Results of the Yale Tardive Dyskinesia Study. Arch Gen Psychiatry 1993; 50(9): 723-733.

7. Miller DD, McEvoy JP, Davis SM, Caroff SN, Saltz $\mathrm{BL}$, Chakos MH, et al. Clinical correlates of tardive dyskinesia in schizophrenia: baseline data from the CATIE schizophrenia trial. Schizophr Res 2005; 80(1): 33-43.
8. Tarsy D, Baldessarini RJ, Tarazi FI. Effects of newer antipsychotics on extrapyramidal function. CNS Drugs. 2002; 16(1): 23-45.

9. Soares-Weiser K, Fernandez HH. Tardive dyskinesia. Semin Neurol 2007; 27(2): 159-69.

10. Higgins JPT, Green S. Cochrane Handbook for Systematic Reviews of Interventions Version 5.0.2: The Cochrane Collaboration.; 2009. Available from: www.cochrane-handbook.org.

11. Hedges LV, Olkin I. Statistical methods for metaanalysis. Orlando: Academic Press; 1985.

12. Morris JA, Gardner MJ. Calculating confidence intervals for relative risks (odds ratios) and standardised ratios and rates. Br Med J (Clin Res Ed). 1988; 296(6632): 13131316.

13. Guy WE. Assessment Manual for Psychopharmacology: Revised (DHEW publication number ADM 76-338). Rockville, MD: US Department of Health, Education and Welfare, Public Health Service, Alcohol, Drug Abuse and Mental Health Administration, NIMH Psychopharmacology Research Branch, Division of extramural Research Programs; 1976. p. 534-537.

14. Chouinard G, Ross-Chouinard A, Annable L, Jones B. Extrapyramidal Symptom Rating Scale. Can J Neurol Sci 1980; 7: 234 .

15. Cassady SL, Thaker GK, Summerfelt A, Tamminga CA. The Maryland Psychiatric Research Center scale and the characterization of involuntary movements. Psychiatry Res 1997; 70(1): 21-37.

16. Simpson GM, Angus JW. A rating scale for extrapyramidal side effects. Acta Psychiatr Scand Suppl 1970; 212: 11-19.

17. Barnes TR. A rating scale for drug-induced akathisia. Br J Psychiatry 1980; 154: 672-676.

18. Overall JE, Gorham DR. The brief psychiatric rating scale. Psychological Reports 1962; 10: 799-812.

19. Kay SR, Fiszbein A, Opler LA. The positive and negative syndrome scale (PANSS) for schizophrenia. Schizophr Bull 1987; 13(2): 261-276.

20. Hall RC. Global assessment of functioning. A modified scale. Psychosomatics 1995; 36(3): 267-275.

21. Folstein MF, Folstein SE, McHugh PR. "Mini-mental state". A practical method for grading the cognitive state of patients for the clinician. J Psychiatr Res 1975; 12(3): 189-198.

22. Hawkins KA, Dean D, Pearlson GD. Alternative forms of the Rey Auditory Verbal Learning Test: a review. Behav Neurol 2004; 15(3-4): 99-107. 
23. Rosvold HE, Delgado JM. The effect on delayed-alternation test performance of stimulating or destroying electrically structures within the frontal lobes of the monkey's brain. J Comp Physiol Psychol 1956; 49(4): 365-372.

24. Stroop JR. Studies of interference in serial verbal reactions. J Exp Psychol 1935; 18: 643-662.

25. Dorevitch A, Kalian M, Shlafman M, Lerner V. Treatment of long-term tardive dyskinesia with vitamin E. Biol Psychiatry 1997; 41(1): 114-116.

26. Sajjad SH. Vitamin E in the treatment of tardive dyskinesia: a preliminary study over 7 months at different doses. Int Clin Psychopharmacol 1998; 13(4): 147-155.

27. Adler LA, Edson R, Lavori P, Peselow E, Duncan E, Rosenthal M, et al. Long-term treatment effects of vitamin $\mathrm{E}$ for tardive dyskinesia. Biol Psychiatry 1998; 43(12): 868-872.

28. Adler LA, Rotrosen J, Edson R, Lavori P, Lohr J, Hitzemann R, et al. Vitamin E treatment for tardive dyskinesia. Veterans Affairs Cooperative Study \#394 Study Group. Arch Gen Psychiatry 1999; 56(9): 836-841.

29. Zhang XY, Zhou DF, Cao LY, Xu CQ, Chen DC, Wu GY. The effect of vitamin $\mathrm{E}$ treatment on tardive dyskinesia and blood superoxide dismutase: a double-blind placebocontrolled trial. J Clin Psychopharmacol 2004; 24(1): 83-86.

30. Hayashi T, Yokota N, Takahashi T, Tawara Y, Nishikawa T, Yano T, et al. Benefits of trazodone and mianserin for patients with late-life chronic schizophrenia and tardive dyskinesia: an add-on, double-blind, placebo-controlled study. Int Clin Psychopharmacol 1997; 12(4): 199-205.

31. Angus S, Sugars J, Boltezar R, Koskewich S, Schneider NM. A controlled trial of amantadine hydrochloride and neuroleptics in the treatment of tardive dyskinesia. J Clin Psychopharmacol 1997; 17(2): 88-91.

32. Pappa S, Tsouli S, Apostolou G, Mavreas V, Konitsiotis S. Effects of amantadine on tardive dyskinesia: a randomized, double-blind, placebo-controlled study. Clin Neuropharmacol 2010; 33(6): 271-275.

33. Cowen MA, Green M, Bertollo DN, Abbott K. A treatment for tardive dyskinesia and some other extrapyramidal symptoms. J Clin Psychopharmacol 1997; 17(3): 190-193.

34. Mosnik DM, Spring B, Rogers K, Baruah S. Tardive dyskinesia exacerbated after ingestion of phenylalanine by schizophrenic patients. Neuropsychopharmacology 1997; 16(2): 136-146.

35. Shamir E, Barak Y, Plopsky I, Zisapel N, Elizur A, Weizman A. Is melatonin treatment effective for tardive dyskinesia? J Clin Psychiatry 2000; 61(8): 556-558.

36. Shamir E, Barak Y, Shalman I, Laudon M, Zisapel N, Tarrasch R, et al. Melatonin treatment for tardive dyskine- sia: a double-blind, placebo-controlled, crossover study. Arch Gen Psychiatry 2001; 58(11): 1049-1052.

37. Lerner V, Miodownik C, Kaptsan A, Cohen H, Matar $\mathrm{M}$, Loewenthal U, et al. Vitamin B(6) in the treatment of tardive dyskinesia: a double-blind, placebo-controlled, crossover study. Am J Psychiatry 2001; 158(9): 1511-1514.

38. Lerner V, Miodownik C, Kaptsan A, Bersudsky Y, Libov I, Sela BA, et al. Vitamin B6 treatment for tardive dyskinesia: a randomized, double-blind, placebo-controlled, crossover study. J Clin Psychiatry 2007; 68(11): 1648-1654.

39. Wonodi I, Adami H, Sherr J, Avila M, Hong LE, Thaker GK. Naltrexone treatment of tardive dyskinesia in patients with schizophrenia. J Clin Psychopharmacol 2004; 24(4): 441-445.

40. Bai YM, Yu SC, Lin CC. Risperidone for severe tardive dyskinesia: a 12-week randomized, double-blind, placebo-controlled study. J Clin Psychiatry 2003; 64(11): 1342-1348.

41. Emsley R, Turner HJ, Schronen J, Botha K, Smit R, Oosthuizen PP. A single-blind, randomized trial comparing quetiapine and haloperidol in the treatment of tardive dyskinesia. J Clin Psychiatry 2004; 65(5): 696-701.

42. Chan HY, Chiang SC, Chang CJ, Gau SS, Chen JJ, $\mathrm{Chen} \mathrm{CH}$, et al. A randomized controlled trial of risperidone and olanzapine for schizophrenic patients with neurolepticinduced tardive dyskinesia. J Clin Psychiatry 2010; 71(9): 1226-1233.

43. Richardson MA, Bevans ML, Read LL, Chao HM, Clelland JD, Suckow RF, et al. Efficacy of the branchedchain amino acids in the treatment of tardive dyskinesia in men. Am J Psychiatry 2003; 160(6): 1117-1124.

44. Emsley R, Niehaus DJ, Koen L, Oosthuizen PP, Turner HJ, Carey P, et al. The effects of eicosapentaenoic acid in tardive dyskinesia: a randomized, placebo-controlled trial. Schizophr Res 2006; 84(1): 112-120.

45. Caroff SN, Walker P, Campbell C, Lorry A, Petro C, Lynch K, et al. Treatment of tardive dyskinesia with galantamine: a randomized controlled crossover trial. J Clin Psychiatry $2007 ; 68(3)$ : 410-415.

46. Libov I, Miodownik C, Bersudsky Y, Dwolatzky T, Lerner V. Efficacy of piracetam in the treatment of tardive dyskinesia in schizophrenic patients: a randomized, doubleblind, placebo-controlled crossover study. J Clin Psychiatry 2007; 68(7): 1031-1037.

47. Woods SW, Saksa JR, Baker CB, Cohen SJ, Tek C. Effects of levetiracetam on tardive dyskinesia: a randomized, double-blind, placebo-controlled study. J Clin Psychiatry 2008; 69(4): 546-554. 
48. Ogunmefun A, Hasnain M, Alam A, Osuala T, Regenold WT. Effect of donepezil on tardive dyskinesia. J Clin Psychopharmacol 2009; 29(1): 102-104.

49. Zhang WF, Tan YL, Zhang XY, Chan RC, Wu HR, Zhou DF. Extract of Ginkgo biloba treatment for tardive dyskinesia in schizophrenia: a randomized, double-blind, placebo-controlled trial. J Clin Psychiatry 2011; 72(5): 615-621.

50. Damier P, Thobois S, Witjas T, Cuny E, Derost P, Raoul S, et al. Bilateral deep brain stimulation of the globus pallidus to treat tardive dyskinesia. Arch Gen Psychiatry 2007; 64(2): 170-176.

51. Spivak B, Mester R, Abesgaus J, Wittenberg N, Adlersberg S, Gonen N, et al. Clozapine treatment for neuroleptic-induced tardive dyskinesia, parkinsonism, and chronic akathisia in schizophrenic patients. J Clin Psychiatry 1997; 58(7): 318-322.

52. Bassitt DP, Louza Neto MR. Clozapine efficacy in tardive dyskinesia in schizophrenic patients. Eur Arch Psychiatry Clin Neurosci 1998; 248(4): 209-211.

53. Kinon BJ, Jeste DV, Kollack-Walker S, Stauffer V, LiuSeifert H. Olanzapine treatment for tardive dyskinesia in schizophrenia patients: a prospective clinical trial with patients randomized to blinded dose reduction periods. Prog Neuropsychopharmacol Biol Psychiatry 2004; 28(6): 985-996.

54. Bai YM, Yu SC, Chen JY, Lin CY, Chou P, Lin CC. Risperidone for pre-existing severe tardive dyskinesia: a 48-week prospective follow-up study. Int Clin Psychopharmacol 2005; 20(2): 79-85.

55. Lerner V, Kaptsan A, Miodownik C, Kotler M. Vitamin B6 in treatment of tardive dyskinesia: a preliminary case series study. Clin Neuropharmacol 1999; 22(4): 241-243.

56. Ondo WG, Hanna PA, Jankovic J. Tetrabenazine treatment for tardive dyskinesia: assessment by randomized videotape protocol. Am J Psychiatry 1999; 156(8): $1279-1281$

57. Sirota P, Mosheva T, Shabtay H, Giladi N, Korczyn $\mathrm{AD}$. Use of the selective serotonin 3 receptor antagonist ondansetron in the treatment of neuroleptic-induced tardive dyskinesia. Am J Psychiatry 2000; 157(2): 287-289.

58. Michael N, Sourgens H, Arolt V, Erfurth A. Severe tardive dyskinesia in affective disorders: treatment with vitamin E and C. Neuropsychobiology 2002; 46 Suppl 1: 28-30.

59. Hardoy MC, Carta MG, Carpiniello B, Cianchetti C, Congia S, D'Errico I, et al. Gabapentin in antipsychotic-induced tardive dyskinesia: results of 1-year follow-up. J Affect Disord 2003; 75(2): 125-130.

60. Richardson MA, Small AM, Read LL, Chao HM, Clelland JD. Branched chain amino acid treatment of tardive dyskinesia in children and adolescents. J Clin Psychiatry 2004; 65(1): 92-96.
61. Bona JR. Treatment of neuroleptic-induced tardive dyskinesia with levetiracetam: a case series. J Clin Psychopharmacol 2006; 26(2): 215-216.

62. Meco G, Fabrizio E, Epifanio A, Morgante F, Valente $\mathrm{M}$, Vanacore $\mathrm{N}$, et al. Levetiracetam in tardive dyskinesia. Clin Neuropharmacol 2006; 29(5): 265-268.

63. Konitsiotis S, Pappa S, Mantas C, Mavreas V. Levetiracetam in tardive dyskinesia: an open label study. Mov Disord 2006; 21(8): 1219-1221.

64. Lee JG, Shin BS, Lee YC, Park SW, Kim YH. Clinical effectiveness of the Kampo medicine kamishoyosan for adjunctive treatment of tardive dyskinesia in patients with schizophrenia: a 16-week open trial. Psychiatry Clin Neurosci 2007; 61(5): 509-514.

65. Miyaoka T, Furuya M, Yasuda H, Hayashida M, Nishida A, Inagaki T, et al. Yi-gan san for the treatment of neuroleptic-induced tardive dyskinesia: an open-label study. Prog Neuropsychopharmacol Biol Psychiatry 2008; 32(3): 761-764.

66. Bouchard RH, Merette C, Pourcher E, Demers MF, Villeneuve J, Roy-Gagnon $\mathrm{MH}$, et al. Longitudinal comparative study of risperidone and conventional neuroleptics for treating patients with schizophrenia. The Quebec Schizophrenia Study Group. J Clin Psychopharmacol 2000; 20(3): 295-304.

67. Purdon SE, Jones BD, Stip E, Labelle A, Addington $\mathrm{D}$, David SR, et al. Neuropsychological change in early phase schizophrenia during 12 months of treatment with olanzapine, risperidone, or haloperidol. The Canadian Collaborative Group for research in schizophrenia. Arch Gen Psychiatry 2000; 57(3): 249-258.

68. Soares-Weiser K, Maayan N, McGrath J. Vitamin E for neuroleptic-induced tardive dyskinesia. Cochrane Database of Systematic Reviews [Internet]. 2010; (2). Available from: http://www.mrw.interscience.wiley.com/cochrane/clsysrev/articles/CD000209/frame.html.

69. Jeste DV, Caligiuri MP. Tardive dyskinesia. Schizophr Bull 1993; 19(2): 303-315.

70. Shale H, Tanner C. Pharmacological options for the management of dyskinesias. Drugs 1996; 52(6): 849-860.

71. Soares-Weiser K, Rathbone J. Neuroleptic reduction and/or cessation and neuroleptics as specific treatments for tardive dyskinesia. Cochrane Database of Systematic Reviews [Internet]. 2006; Available from:

http://www.mrw.interscience.wiley.com/cochrane/clsysrev/ articles/CD000459/frame.html.

72. Bender DA. Non-nutritional uses of vitamin B6. Br J Nutr 1999; 81(1): 7-20. 
73. Leung JG, Breden EL. Tetrabenazine for the treatment of tardive dyskinesia. Ann Pharmacother 45(4): 525-531.

74. Wolf MA, Yassa R, Llorca PM. Neuroleptic-induced movement disorders: historical perspectives. Encephale 1993; 19(6): 657-661.

75. Casey DE. Tardive dyskinesia: pathophysiology and animal models. J Clin Psychiatry 2000; 61 Suppl 4: 5-9.
Corresponding author:

Dr Michael Alimi

Clinique Psychiatrique Universitaire

CHRU de Tours

Tours, France

E-mail: michael.alimi@ymail.com 